\title{
Majorization-Minimization Algorithms for Analog Beamforming with Large-Scale Antenna Arrays
}

\author{
Aakash Arora, Christos G. Tsinos, Bhavani Shankar Mysore R, Symeon Chatzinotas and Björn Ottersten \\ Interdisciplinary Centre for Security, Reliability and Trust (SnT), University of Luxembourg \\ email address: \{aakash.arora, christos.tsinos, bhavani.shankar, symeon.chatzinotas, bjorn.ottersten\}@uni.lu
}

\begin{abstract}
Beamforming with large-scale antenna arrays (LSAA) is one of the predominant operations in designing wireless communication systems. However, the implementation of a fully digital system significantly increases the number of required radio-frequency $(\mathrm{RF})$ chains, which may be prohibitive. Thus, analog beamforming based on a phase-shifting network driven by a variable gain amplifier (VGA) is a potential alternative technology. In this paper, we cast the beamforming vector design problem as a beampattern matching problem, with an unknown power gain. This is formulated as a unit-modulus leastsquares (ULS) problem where the optimal gain of the VGA is also designed in addition to the beamforming vector. We also consider a scenario where the receivers have the additional processing capability to adjust the phases of the incoming signals to mitigate specular multipath components. We propose efficient majorization-minimization (MM) based algorithms with convergence guarantees to a stationary point for solving both variants of the proposed ULS problem. Numerical results verify the effectiveness of the proposed solution in comparison with the existing state-of-the-art techniques.
\end{abstract}

Index Terms-Analog beamforming, large-scale antenna arrays, majorization-minimization, MM, beampattern matching.

\section{INTRODUCTION}

In mmWave systems, a large-scale antenna array (LSAA) is used to achieve beamforming gains and to combat severe propagation losses [1], [2]. However, the fully-digital implementation of such a system requires as many radio frequency (RF) chains as the number of antenna elements. Consequently, hardware cost and power consumption may substantially increase. These drawbacks limit the applicability of a system with an LSAA, thus a potential candidate is to employ beamforming networks based only on simple analog components, like phase shifters (PS). Such systems only manipulate the phase of each beamforming component while keeping the magnitude constant; this, coupled with a constant beamformer magnitude, eases their practical implementation.

The analog phase-only beamforming problem has been studied in the past [3]-[7], where the optimal beamforming vector is designed by optimizing a performance metric subject to unit-modulus constraints, e.g., by solving the signal-tointerference-plus-noise ratio (SINR) maximization problem [3]. Due to unit-modulus constraints, the resulting optimization problem is nonconvex and in general NP-hard. The referenced work presents an algorithm based on the conjugate gradient

This work is supported by the National Research Fund (FNR), Luxembourg under the AFR-PPP grant for Ph.D. project SPASAT (Ref.: 11607283), the CORE-PPP project PROSAT, ECLECTIC, CI-PHY and DISBuS. and Newton's method to compute the beamforming weights. A gradient search algorithm is presented in [4], which parameterizes the unit-modulus constraint to adaptively adjust the phases of the entries of the beamforming vector. Receive beamforming is studied in [5] and the optimal beamforming weights are designed by minimizing the mean squared error (MSE) between the array output and the desired signal. For null steering, a position-perturbation technique is presented in [6].

Recently, the analog beamforming problem is considered in [7]. The referenced work designs the analog beamforming weights by minimizing the beampattern matching error in a least-square sense, subject to the unit-modulus constraints. The beampattern matching error is the difference between the desired and the designed beampatterns. This problem is termed as a unit-modulus least squares (ULS) problem [7]. The closely related problem of unimodular radar sequence (or code) design also arises in several active sensing applications [8]. The ULS problem is also nonconvex and shown to be NP-hard [9]. In the literature, an approximate solution to the ULS problem is found by employing the semidefinite relaxation (SDR), which increases the dimensionality of the search space from $M$ to $M^{2}$. Therefore, a large number of design parameters need to be optimized, resulting in increased memory usage and storage requirements. Another disadvantage with SDR is that it empirically returns a rank-1 solution, but in cases when the solution is not rank-1, an appropriate randomization technique should be employed [9], [10]; this further increases the time overhead. Therefore, in order to keep the computational complexity low, gradient-projection (GP) based algorithms are proposed in [7]. Another recent work proposed an alternating direction method of multipliers (ADMM) based algorithm for beamforming in the context of sensor networks [11].

Following similar lines, in this paper, we propose efficient analog beamforming algorithms with convergence guarantees to solve the variants of the ULS problem. Specifically, we consider the analog beamforming architecture with a common variable gain amplifier (VGA) driving the phase-shifting network. Therefore, in addition to the beamforming vector design, we also consider the gain of the VGA as a design variable. We propose to design these variables by solving the beampattern matching problem. This scenario is modeled as the constantmodulus least squares problem, where the VGA gain is modeled as the magnitude of the beamforming vector. To solve this 
problem, we propose a block majorization-minimization (MM) (or more precisely an alternating majorization-minimization (MM)) [12] based algorithm.

We further consider the case when the receivers employ additional processing to mitigate the specular multipath components. This results in additional degrees of freedom in the aforementioned problem. Again, we employ the alternating MM framework to solve the problem.

We show that the proposed algorithms have convergence guarantees to a stationary point. We provide numerical simulations to demonstrate the effectiveness of the proposed algorithms to the ones proposed in [7], resulting in a much better beampattern approximation.

\section{Problem Fomrulation}

We consider two analog beamforming architectures (i) scaled analog beamforming (SAB) and (ii) phase-corrected analog beamforming (PSAB) shown in Fig. 1. We then formulate the analog beamforming problem for these architectures.

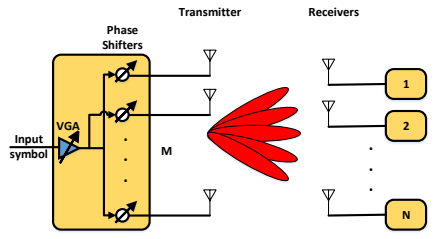

(a) SAB.

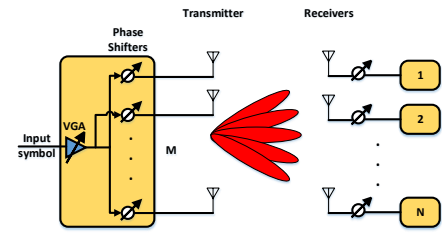

(b) PSAB.
Fig. 1: Analog Beamforming Architectures.

\section{A. SAB Architecture}

A transmitter equipped with a phase-shifting network driven by a common VGA serving multiple single-antenna users is considered, as shown in Fig. 1a. The use of a common VGA for all branches motivates the term SAB architecture. Herein, we consider a uniform linear array (ULA) with $M$ antenna elements and a spacing $d=\frac{\lambda}{2}$ at the transmitter, where $\lambda$ represents the wavelength of operation. The array transmits the same information to the users, therefore, this a broadcast beamforming scenario [13]. The array response of a ULA in a direction $\theta_{i}$ is modeled as,

$$
\mathbf{a}\left(\theta_{i}\right)=\left[\begin{array}{lllll}
1 & e^{-j \theta_{i}} & e^{-j 2 \theta_{i}} & \ldots & e^{-j(M-1) \theta_{i}}
\end{array}\right]^{T} .
$$

We consider an uniform discretization of the angular space into $N$ points as $\boldsymbol{\theta}=\left[0, \frac{2 \pi}{N}, \frac{4 \pi}{N}, \ldots, \frac{2(N-1) \pi}{N}\right]^{T}$. Then, the array response from these directions can be written in matrix form as $\mathbf{A}(\boldsymbol{\theta})=\left[\mathbf{a}\left(\theta_{1}\right), \mathbf{a}\left(\theta_{2}\right), \ldots, \mathbf{a}\left(\theta_{N}\right)\right]^{H}$, where $\theta_{i}$ represents the $i$-th element of $\boldsymbol{\theta}$. For notational convenience, henceforth, we write $\mathbf{A}(\boldsymbol{\theta})$ and $\mathbf{a}\left(\theta_{i}\right)$ as $\mathbf{A}$ and $\mathbf{a}_{i}$, respectively.

The beampattern in the direction $\theta_{i}$ takes the form $y_{i}=$ $\mathbf{a}\left(\theta_{i}\right)^{H} \tilde{\mathbf{w}}$, where $\tilde{\mathbf{w}}$ is the beamforming vector to be designed. Therefore, the least-squares beampattern matching problem considering the requirements in all $\left\{\theta_{i}\right\}$ is formulated as,

$$
\begin{gathered}
\mathcal{P}_{1}: \min _{r \in \mathbb{R}, \tilde{\mathbf{w}}}\|\mathbf{y}-\mathbf{A}(\boldsymbol{\theta}) \tilde{\mathbf{w}}\|_{2}^{2} \\
\text { subject to } \tilde{\mathbf{w}} \in \mathcal{A}^{\prime},
\end{gathered}
$$

where $\mathbf{y}$ is an $N$-dimensional vector denoting the desired response along the directions represented by the elements of vector $\boldsymbol{\theta}, \mathcal{A}^{\prime}=\left\{\tilde{\mathbf{w}} \in \mathbb{C}^{M \times 1}|| \mathbf{w}(i) \mid=r, \forall i \in\{1,2, \ldots, M\}\right\}$ and the variable $r$ models the gain introduced by the VGA. The elements of $\tilde{\mathbf{w}}$ are constrained to be constant modulus, thus representing phase-only beamforming.

We reformulate $\mathcal{P}_{1}$ in a compact manner as,

$$
\begin{gathered}
\mathcal{P}_{2}: \min _{s \in \mathbb{C}, \mathbf{w}}\|\mathbf{y}-s \mathbf{A}(\boldsymbol{\theta}) \mathbf{w}\|_{2}^{2} \\
\text { subject to } \mathbf{w} \in \mathcal{A},
\end{gathered}
$$

where $\mathcal{A}=\left\{\left.\mathbf{w} \in \mathbb{C}^{M \times 1}|| \mathbf{w}(i)\right|^{2}=1, \forall i \in\{1,2, \ldots, M\}\right\}$. The constant modulus constraint in $\mathcal{P}_{1}$ is equivalently replaced by an unconstrained complex factor $s$ multiplying $\mathbf{w}$. The beamformers are now constrained to have unit-modulus entries.

Problem $\mathcal{P}_{2}$ is nonconvex and a joint solution in $s$ and $\mathbf{w}$ is intractable. But the objective function is partially convex: it is convex in one variable given the other. This observation naturally leads to the development of a block-MM (or more precisely speaking an alternating-MM) algorithm, which also works on the MM principle for each block [12]. However, in the case of block-MM, the optimization problem with respect to each block is approximated using a majorizing function such that the approximate problem admits a closed-form solution. For more information, one may refer to [12], [14]-[17] and references therein. As mentioned earlier, our approach is different from block-MM as we do not consider a majorizing function for all the variables; instead, we approximate the problem only with respect to those blocks for which the subproblem is not easy to minimize.

Assuming $\mathbf{w}$ to be given, it can be readily seen that the minimization problem to solve for variable $s$ admits the following closed-form solution [7],

$$
s=\frac{\mathbf{w}^{H} \mathbf{A}^{H} \mathbf{y}}{\|\mathbf{A w}\|_{2}^{2}} .
$$

Now, considering $s$ to be given, the minimization problem with respect to $\mathbf{w}$ can be written as,

$$
\begin{gathered}
\mathcal{P}_{3}: \quad \min _{\mathbf{w}} \quad f(\mathbf{w})=\|\mathbf{y}-s \mathbf{A w}\|_{2}^{2} \\
\text { subject to } \mathbf{w} \in \mathcal{A} .
\end{gathered}
$$

The objective in problem $\mathcal{P}_{3}$ takes the form,

$$
f(\mathbf{w})=\mathbf{y}^{H} \mathbf{y}-2 \operatorname{Re}\left(s \mathbf{y}^{H} \mathbf{A} \mathbf{w}\right)+|s|^{2} \mathbf{w}^{H} \mathbf{A}^{H} \mathbf{A} \mathbf{w} .
$$

It is easy to see that the last term of the objective function in (5) is convex in w. Therefore, we approximate this term using the Lemma II.1

Lemma II.1. The quadratic function of the form $\mathbf{a}^{H} \mathbf{S a}$, with $\mathbf{S}$ being a Hermitian matrix is majorized by $\mathbf{a}^{H} \mathbf{T a}+$ $2 \operatorname{Re}\left(\mathbf{a}^{H}(\mathbf{S}-\mathbf{T}) \mathbf{a}_{k}\right)+\mathbf{a}_{k}^{H}(\mathbf{T}-\mathbf{S}) \mathbf{a}_{k}$ at the point $\mathbf{a}_{k}$, where $\mathbf{T}$ is a Hermitian matrix such that $\mathbf{T} \succeq \mathbf{S}$.

Lemma II.1 can be easily proven using second order Taylor expansion and subsequently replacing the $\mathbf{S}$ by another Hermitian matrix $\mathbf{T}$ such that $\mathbf{T} \succeq \mathbf{S}$, [14], [17]. Using Lemma 




II.1 with $\mathbf{T}=\lambda_{\max }(\tilde{\mathbf{P}}) \mathbf{I}$ where $\mathbf{I}$ is a $M \times M$ identity matrix and $\tilde{\mathbf{P}}=|s|^{2} \mathbf{A}^{H} \mathbf{A}$, the quadratic term in (5) is majorized as,

$$
\begin{aligned}
\mathbf{w}^{H} \tilde{\mathbf{P}} \mathbf{w} \leq & \lambda_{\max }(\tilde{\mathbf{P}}) \mathbf{w}^{H} \mathbf{w} \\
& +2 \operatorname{Re}\left(\mathbf{w}^{H}\left(\tilde{\mathbf{P}}-\lambda_{\max }(\tilde{\mathbf{P}}) \mathbf{I}\right) \mathbf{w}_{k}\right)+\mathbf{w}_{k}^{H} \tilde{\mathbf{P}} \mathbf{w}_{k},
\end{aligned}
$$

where $\mathbf{w}_{k}$ is the solution available $k$-th iteration. It is easy to see that the first term in (6) equals $\lambda_{\max }(\tilde{\mathbf{P}}) M$, due to the unit modulus property of the beamfomring vector $\mathbf{w}$. The third term is independent of $\mathbf{w}$. Neglecting the constant terms in (5) and (6), the overall majorizer of the objective function in (5) can be expressed as,

$$
-2 \operatorname{Re}\left(s \mathbf{y}^{H} \mathbf{A w}\right)+2 \operatorname{Re}\left(\mathbf{w}^{H}\left(\tilde{\mathbf{P}}-\lambda_{\max }(\tilde{\mathbf{P}}) \mathbf{I}\right) \mathbf{w}_{k}\right) .
$$

The resulting majorized problem can be formulated as,

$$
\begin{aligned}
\mathcal{P}_{4}: \min _{\mathbf{w}}-2\left[\operatorname{Re}\left(s^{*} \mathbf{w}^{H} \mathbf{A}^{H} \mathbf{y}\right)-\operatorname{Re}\left(\mathbf{w}^{H}|s|^{2}(\mathbf{P}-\beta \mathbf{I}) \mathbf{w}_{k}\right)\right] \\
\quad \text { subject to } \mathbf{w} \in \mathcal{A},
\end{aligned}
$$

where $\mathbf{P}=\mathbf{A}^{H} \mathbf{A}$ and $\beta=\lambda_{\max }(\mathbf{P})$. Problem $\mathcal{P}_{4}$ can be further reformulated as,

$$
\begin{gathered}
\mathcal{P}_{5}: \min _{\mathbf{w}}\left\|\mathbf{w}-\left(s^{*} \mathbf{A}^{H} \mathbf{y}-|s|^{2}(\mathbf{P}-\beta \mathbf{I}) \mathbf{w}_{k}\right)\right\|_{2}^{2} \\
\text { subject to } \mathbf{w} \in \mathcal{A} .
\end{gathered}
$$

Now, $\mathcal{P}_{5}$ readily admits the following closed-form solution,

$$
\mathbf{w}=e^{j \arg \left(\mathbf{c}_{k}\right)},
$$

where $\mathbf{c}_{k}=s^{*} \mathbf{A}^{H} \mathbf{y}-|s|^{2}(\mathbf{P}-\beta \mathbf{I}) \mathbf{w}_{k}$. The overall methodology is presented in Algorithm 1. This algorithm is based on the alternating-MM framework, where the sub-problems with respect only those variables (here, w) are approximated for which direct minimization is difficult.

Theorem II.2. Let $\left\{\mathbf{w}_{k}, s_{k}\right\}$ be the sequence of outputs generated by Algorithm 1. Then this solution sequence converges to the set of $K K T$ points $(\mathcal{K})$ of problem $\mathcal{P}_{5}$.

Proof. For brevity, the proof is omitted and will be available in a future journal submission.

\section{B. PSAB Architecture}

Until now, the beampattern matching problem was mainly considered around transmit beamforming. As already considered in [7], in many cases, the receiver is also required to control the phase response of the incoming signal by using an analog phase-shifting network as shown in Fig. 1b. This entails additional degrees of freedom to enhance the beamforming performance.

Taking care into account scaling and phase-correction, the beamforming design problem can be formulated as,

$$
\begin{gathered}
\mathcal{P}_{6}: \min _{\substack{s \in \mathbb{C}, \mathbf{w}, \mathbf{u} \\
\text { subject to } \mathbf{w} \in \mathcal{A}, \mathbf{u} \in \mathcal{A},}} h(s, \mathbf{w}, \mathbf{u})=\|\mathbf{y} \circ \mathbf{u}-s \mathbf{A} \mathbf{w}\|_{2}^{2}
\end{gathered}
$$

where $\circ$ denotes the element-wise product between two vectors or matrices. Further, $\mathbf{u}$ is a vector having unit-modulus entries and thus models the phase correction term. The problem $\mathcal{P}_{6}$ is also nonconvex; we, therefore, adopt the alternating-MM framework again for finding a solution. Considering variables $\mathbf{w}$ and $\mathbf{u}$ to be given, the problem with respect to variables $s$ admits the following closed-form solution,

$$
s=\frac{\mathbf{w}^{H} \mathbf{A}^{H} \mathbf{Y u}}{\|\mathbf{A} \mathbf{w}\|_{2}^{2}},
$$

where $\mathbf{Y}=\operatorname{diag}(\mathbf{y})$ is a diagonal matrix with entries of $\mathbf{y}$. With regards to the variable $\mathbf{u}$, upon expanding the objective function and ignoring the constant terms, we obtain the following problem,

$$
\begin{gathered}
\mathcal{P}_{7}: \min _{\mathbf{u}}-2 \operatorname{Re}\left(s \mathbf{u}^{H} \mathbf{Y}^{H} \mathbf{A w}\right) \\
\text { subject to } \mathbf{u} \in \mathcal{A} .
\end{gathered}
$$

Problem $\mathcal{P}_{7}$ can be further reformulated as,

$$
\begin{gathered}
\mathcal{P}_{8}: \min _{\mathbf{u}}\left\|\mathbf{u}-s \mathbf{Y}^{H} \mathbf{A w}\right\|_{2}^{2} \\
\text { subject to } \mathbf{u} \in \mathcal{A} .
\end{gathered}
$$

It is straightforward to see that problem $\mathcal{P}_{8}$ admits the following closed-form solution,

$$
\mathbf{u}=e^{j \arg \left(s \mathbf{Y}^{H} \mathbf{A w}\right)} .
$$

With regards to the variable $\mathrm{w}$, the solution can be found by majorizing the objective function using Lemma II.1, similar to the one obtained for $\mathcal{P}_{3}$. After ignoring the constant terms, we arrive at the following problem,

$$
\begin{aligned}
& \mathcal{P}_{9}: \min _{\mathbf{w}}-2 \operatorname{Re}\left(\mathbf{w}\left(s^{*} \mathbf{A}^{H} \mathbf{Y} \mathbf{u}-\mathbf{Q} \mathbf{w}_{k}\right)\right) \\
& \text { subject to } \mathbf{w} \in \mathcal{A} .
\end{aligned}
$$

where $\mathbf{Q}=|s|^{2}\left(\mathbf{P}-\lambda_{\max }(\mathbf{P}) \mathbf{I}\right)$ and $\mathbf{w}_{k}$ is the solution at $k$-th iteration. Problem $\mathcal{P}_{9}$ is equivalently reformulated as,

$$
\begin{gathered}
\mathcal{P}_{10}: \min _{\mathbf{w}}\left\|\mathbf{w}-\left(s^{*} \mathbf{A}^{H} \mathbf{Y} \mathbf{u}-\mathbf{Q} \mathbf{w}_{k}\right)\right\|_{2}^{2} \\
\text { subject to } \mathbf{w} \in \mathcal{A} .
\end{gathered}
$$

It is readily seen that $\mathcal{P}_{10}$ admits the following closed-form solution,

$$
\mathbf{w}=e^{j \arg \left(\tilde{\mathbf{w}}_{k}\right)},
$$

where $\tilde{\mathbf{w}}_{k}=s^{*} \mathbf{A}^{H} \mathbf{Y u}-\mathbf{Q w}_{k}$. The complete algorithm summarizing the steps is presented in Algorithm 2. Similar, to Algorithm 1, the convergence of the sequence $\left\{s_{k}, \mathbf{u}_{k}, \mathbf{w}_{k}\right\}$ generated by Algorithm 2 to a stationary point of problem $\mathcal{P}_{6}$ can be established. 

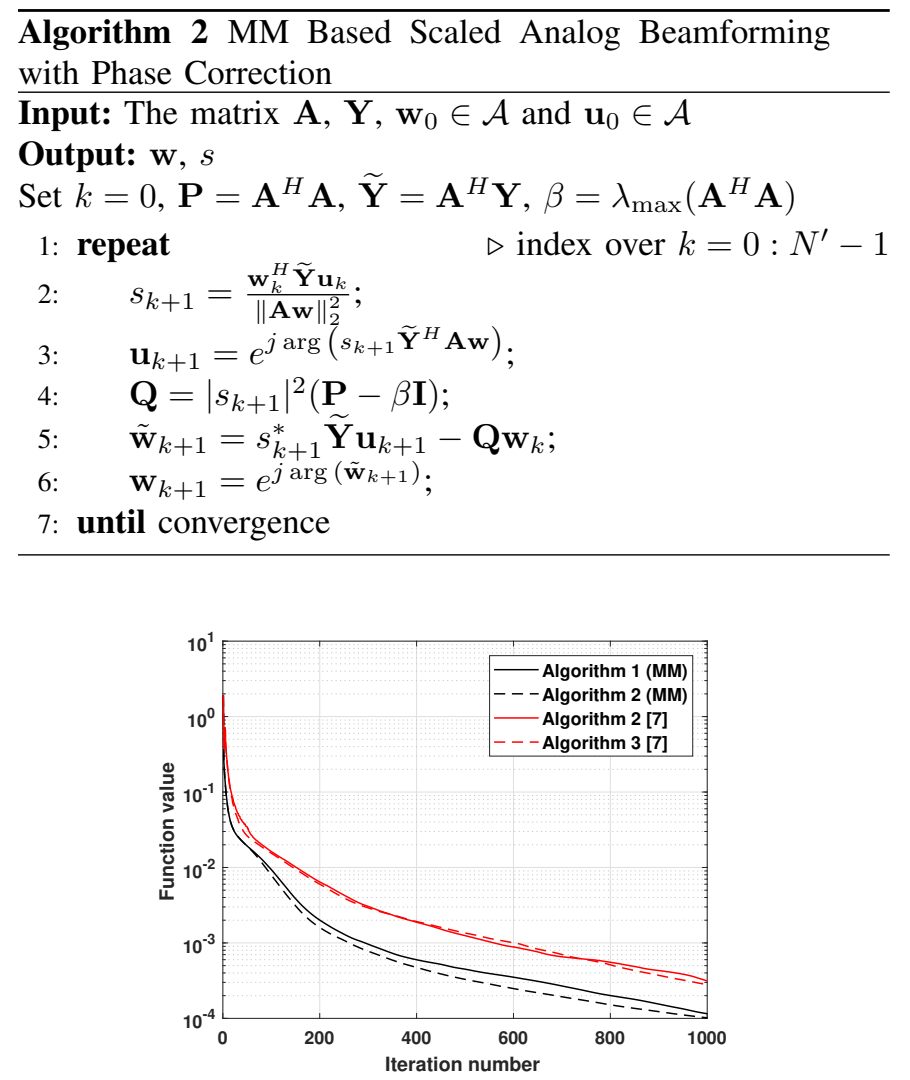

Fig. 2: Objective function variation with the number of iterations, $N=36, M=100$.

\section{Simulation Results}

We consider the objective functions of all the problems as performance metrics of the proposed algorithms and compare them to the ones proposed in [7]. Specifically, we analyze the evolution of objective function with the number of iterations. We consider $M=100$ and discretize the angular space with $N=36$ points and generate $\mathbf{y}$ according to (12) below,

$$
y_{i}= \begin{cases}1 & \text { if } i \in \mathcal{I}, \\ 0 & \text { otherwise }\end{cases}
$$

where $\mathcal{I} \in[1, N]$ denotes the index set for non-zero entries of vector $\mathbf{y}$ with cardinality of $\operatorname{card}(\mathcal{I})=K$. All the algorithms are initialized identically. For fairness, we randomly choose $K=2$ entries for $\mathcal{I}$ from $[1, N]$, and select the corresponding $K$ angles from $\boldsymbol{\theta}$. We then obtain $\mathbf{y}$ according to (12).

Fig. 2 shows the comparison of Algorithm 1 and Algorithm 2 to those proposed in [7] to solve problem $\mathcal{P}_{2}$ and $\mathcal{P}_{6}$, respectively. It is observed that the proposed algorithms have much better beampattern approximation accuracy than those from [7].

As mentioned earlier, in many cases, instead of drawing pencil beams, we may be interested in a sector of angles. For example, in multicasting scenarios where users in one group receive the same information. Therefore, we consider a different arrangement to study the performance of the proposed

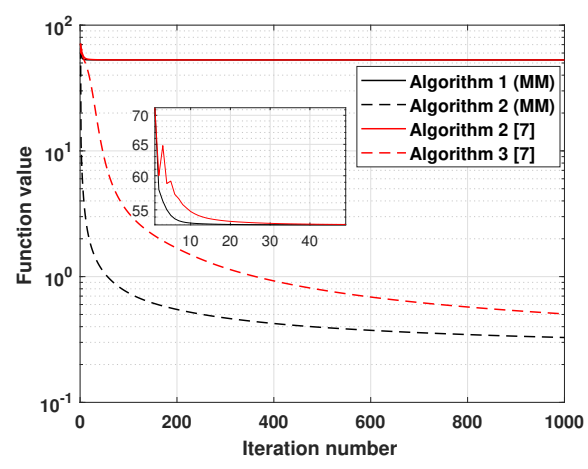

Fig. 3: Objective function variation with the number of iterations, $N=150, M=200$.

TABLE I: Average running time comparison

\begin{tabular}{|l|c|c|c|}
\hline \multicolumn{4}{|c|}{ Average running time (seconds) } \\
\hline Algorithms & $M=50$ & $M=100$ & $M=200$ \\
\hline Algorithm 1 & $4.6 \times 10^{-5}$ & $1.2 \times 10^{-4}$ & $3.8 \times 10^{-4}$ \\
Algorithm 2 [7] & $5.8 \times 10^{-5}$ & $1.3 \times 10^{-5}$ & $3.8 \times 10^{-4}$ \\
Algorithm 2 & $2.0 \times 10^{-4}$ & $3.6 \times 10^{-4}$ & $7.0 \times 10^{-4}$ \\
Algorithm 3 [7] & $2.3 \times 10^{-4}$ & $4.1 \times 10^{-4}$ & $8.4 \times 10^{-4}$ \\
\hline
\end{tabular}

algorithms. Specifically, we consider, $N=150, M=200$ and $\mathcal{I}=\{1,2, \ldots, 18,55,56, \ldots, 90,127,128, \ldots, 144\}$. For this case, Fig. 3 shows the comparison of the proposed algorithms with the ones in [7]. It can be easily seen that the proposed algorithms show faster convergence and result in significantly improved beampattern approximation. We also want to highlight the fact that the performance of the proposed Algorithm 1 and Algorithm 2 from [7] is significantly degraded (but our algorithm still has better performance) in the sectored beamforming scenario, whereas Algorithm 2 results in much better beampattern approximation due to the additional degrees of freedom available at the receiver's side.

In order to get insights about the execution time of the algorithms, Table I shows the average running times. The first two rows consider the pencil beams scenario whereas the last two rows consider the sectored beam scenario. The stopping criteria chosen for the algorithms is either $\frac{\left\|\mathbf{w}_{k}-\mathbf{w}_{k-1}\right\|_{2}}{\left\|\mathbf{w}_{k}\right\|_{2}} \leq$ $10^{-6}$ or 5000 iterations. It is readily seen that the proposed algorithms require lower CPU time to converge with lesser beampattern matching error (as observed from Figs. 2 and 3).

\section{CONCLusions}

In this paper, we considered two analog beamforming problems namely, scaled analog beamforming and phasecorrected analog beamforming. We proposed efficient algorithms based on the majorization-minimization framework with convergence guarantees to a stationary point for solving these problems. Simulation results demonstrated that the proposed algorithms require lesser time to converge with a better objective value in comparison to the algorithms existing in the literature. These result in a faster and better beampattern approximation method. 


\section{REFERENCES}

[1] A. Alkhateeb, O. El Ayach, G. Leus, and R. W. Heath, "Channel estimation and hybrid precoding for millimeter wave cellular systems," IEEE J. Sel. Topics Signal Process., vol. 8, no. 5, pp. 831-846, Oct 2014.

[2] O. E. Ayach, S. Rajagopal, S. Abu-Surra, Z. Pi, and R. W. Heath, "Spatially sparse precoding in millimeter wave MIMO systems," IEEE Trans. Wireless Commun., vol. 13, no. 3, pp. 1499-1513, March 2014.

[3] S. T. Smith, "Optimum phase-only adaptive nulling," IEEE Trans. Signal Process., vol. 47, no. 7, pp. 1835-1843, July 1999.

[4] P. Thompson, "Adaptation by direct phase-shift adjustment in narrowband adaptive antenna systems," IEEE Trans. Antennas Propag., vol. 24, no. 5, pp. 756-760, Sep. 1976.

[5] C. Baird and G. Rassweiler, "Adaptive sidelobe nulling using digitally controlled phase-shifters," IEEE Trans. Antennas Propag., vol. 24, no. 5, pp. 638-649, Sep. 1976.

[6] T. H. Ismail and M. M. Dawoud, "Null steering in phased arrays by controlling the element positions," IEEE Trans. Antennas Propag., vol. 39, no. 11, pp. 1561-1566, Nov 1991.

[7] J. Tranter, N. D. Sidiropoulos, X. Fu, and A. Swami, "Fast unit-modulus least squares with applications in beamforming," IEEE Trans. Signal Process., vol. 65, no. 11, pp. 2875-2887, June 2017.

[8] M. Soltanalian and P. Stoica, "Designing unimodular codes via quadratic optimization," IEEE Trans. Signal Process., vol. 62, no. 5, pp. 12211234, March 2014.

[9] S. Zhang and Y. Huang, "Complex quadratic optimization and semidefinite programming," SIAM Journal on Optimization, vol. 16, no. 3, pp. 871-890, 2006. [Online]. Available: https://doi.org/10.1137/04061341X

[10] Z. Luo, W. Ma, A. M. So, Y. Ye, and S. Zhang, "Semidefinite relaxation of quadratic optimization problems," IEEE Signal Process. Mag., vol. 27, no. 3, pp. 20-34, May 2010.

[11] C. G. Tsinos and B. Ottersten, "An efficient algorithm for unit-modulus quadratic programs with application in beamforming for wireless sensor networks," IEEE Signal Process. Lett., vol. 25, no. 2, pp. 169-173, Feb 2018.

[12] M. Razaviyayn, M. Hong, and Z. Luo, "A unified convergence analysis of block successive minimization methods for nonsmooth optimization," SIAM Journal on Optimization, vol. 23, no. 2, pp. 1126-1153, 2013. [Online]. Available: https://doi.org/10.1137/120891009

[13] M. Alodeh, D. Spano, A. Kalantari, C. G. Tsinos, D. Christopoulos, S. Chatzinotas, and B. Ottersten, "Symbol-level and multicast precoding for multiuser multiantenna downlink: A state-of-the-art, classification, and challenges," IEEE Commun. Surveys Tuts., vol. 20, no. 3, pp. 17331757, thirdquarter 2018.

[14] D. R. Hunter and K. Lange, "A tutorial on MM algorithms," The American Statistician, vol. 58, no. 1, pp. 30-37, 2004. [Online]. Available: https://doi.org/10.1198/0003130042836

[15] K. Lange, MM Optimization Algorithms. SIAM, 2016, vol. 147.

[16] Y. Sun, P. Babu, and D. P. Palomar, "Majorization-Minimization algorithms in signal processing, communications, and machine learning," IEEE Trans. Signal Process., vol. 65, no. 3, pp. 794-816, Feb 2017.

[17] J. Song, P. Babu, and D. P. Palomar, "Optimization methods for designing sequences with low autocorrelation sidelobes," IEEE Trans. Signal Process., vol. 63, no. 15, pp. 3998-4009, Aug 2015. 